ARTICLE

\title{
Mesocortical BDNF signaling mediates antidepressive-like effects of lithium
}

\author{
Di Liu' ${ }^{1,2}$, Qian-Qian Tang ${ }^{1,2}$, Di Wang ${ }^{1,2}$, Su-Pei Song ${ }^{1,2}$, Xiao-Na Yang ${ }^{1,2}$, Su-Wan Hu ${ }^{1,2}$, Zhi-Yong Wang ${ }^{1,2}$, Zheng Xu ${ }^{1,2}$, He Liu ${ }^{1,2}$,
} Jun-Xia Yang ${ }^{1,2}$, Sarah E. Montgomery ${ }^{3,4}$, Hongxing Zhang ${ }^{1,2}$, Ming-Hu Han ${ }^{3,4}$, Hai-Lei Ding ${ }^{1,2}$ and Jun-Li Cao ${ }^{1,2,5}$

Lithium has been used to treat major depressive disorder, yet the neural circuit mechanisms underlying this therapeutic effect remain unknown. Here, we demonstrated that the ventral tegmental area (VTA) dopamine (DA) neurons that project to the medial prefrontal cortex (mPFC), but not to nucleus accumbens (NAc), contributed to the antidepressive-like effects of lithium. Projectionspecific electrophysiological recordings revealed that high concentrations of lithium increased firing rates in mPFC-, but not NAc-, projecting VTA DA neurons in mice treated with chronic unpredictable mild stress (CMS). In parallel, chronic administration of highdose lithium in CMS mice restored the firing properties of MPFC-projecting DA neurons, and also rescued CMS-induced depressivelike behaviors. Nevertheless, chronic lithium treatment was insufficient to change the basal firing rates in NAc-projecting VTA DA neurons. Furthermore, chemogenetic activation of mPFC-, but not NAc-, projecting VTA DA neurons mimicked the antidepressivelike effects of lithium in CMS mice. Chemogenetic downregulation of VTA-mPFC DA neurons' firing activity abolished the antidepressive-like effects of lithium in CMS mice. Finally, we found that the antidepressant-like effects induced by high-dose lithium were mediated by BNDF signaling in the mesocortical DA circuit. Together, these results demonstrated the role of mesocortical DA projection in antidepressive-like effects of lithium and established a circuit foundation for lithium-based antidepressive treatment.

Neuropsychopharmacology (2020) 45:1557-1566; https://doi.org/10.1038/s41386-020-0713-0

\section{INTRODUCTION}

In addition to bipolar disorders, lithium is also used to treat major depressive disorder with promising efficacies [1-4], including therapy for treatment-resistant depression $[5,6]$ and augmentation of antidepressants [7,8]. Through pharmacological, molecular, and genetic methods, numerous studies have explored that several molecular targets including glycogen synthase kinase 3, CREB, mTOR, Wnt, and inositol signaling pathways may mediate lithium's therapeutic efficacy [9-12]. However, the neural circuit and cellular mechanisms underlying the antidepressive-like effects of lithium remain poorly understood.

In rodent models, depressive-like behaviors are causally associated with the abnormal neuronal activity of mesocorticolimbic ventral tegmental area (VTA) dopamine (DA) neurons [13-18]. The mesocorticolimbic DA circuits, which are composed of two separate populations of VTA DA neurons that project to the medial prefrontal cortex (mPFC) and the nucleus accumbens (NAc), respectively, show considerable functional heterogeneity [19-22]. For instance, in the social defeat stress model of depression, NAc-projecting VTA DA neurons exhibit an increased firing rate while those projecting to MPFC show a dramatically decreased firing activity $[14,15]$. Optogenetic inhibition of the VTA-NAc projection induces resilience, whereas inhibition of the
VTA-mPFC projection promotes susceptibility to social defeat stress $[14,15]$. Inhibition of the DA neuron firing by expression of KCNQ3 (potassium voltage-gated channel subfamily Q member 3 ) in the VTA-NAc pathway, but not in the VTA-mPFC pathway, results in a reversal of depressive-like behaviors in susceptible mice to social defeat [23]. These studies demonstrate the differential roles of the mesocorticolimbic DA circuits in the development of depression-like behavioral abnormalities. Previous studies have shown that the mesocorticolimbic DA systems are regulated by lithium treatment. For example, lithium administration decreases the spontaneous firing rates of VTA DA neurons in a mouse model in which circadian rhythm and sleep are impaired [24]. Furthermore, lithium administration ameliorates manic-like phenotypes by restoring the homeostasis in VTA DA neurons [25-27]. These results lead to our hypothesis that the mesocorticolimbic DA circuits may be the primary targets of lithium to produce antidepressive-like effects.

Molecular studies have implicated that the brain-derived neurotrophic factor (BDNF), which is known to play roles in neuronal survival, neuroplasticity, and depression [28-31], may underlie the therapeutic actions of lithium. Consistent with these investigations, the antidepressive-like effects of lithium treatments are associated with activation of BDNF signaling and increased

\footnotetext{
${ }^{1}$ Jiangsu Province Key Laboratory of Anesthesiology, Xuzhou Medical University, Xuzhou 221004 Jiangsu, China; ${ }^{2}$ Jiangsu Province Key Laboratory of Anesthesia and Analgesia Application Technology, Xuzhou Medical University, Xuzhou 221004 Jiangsu, China; ${ }^{3}$ Department of Pharmacological Sciences, Institute for Systems Biomedicine, Icahn School of Medicine at Mount Sinai, New York, NY 10029-6574, USA; ${ }^{4}$ Fishberg Department of Neuroscience, Friedman Brain Institute, Icahn School of Medicine at Mount Sinai, New York, NY 10029-6574, USA and ${ }^{5}$ Department of Anesthesiology, The Affiliated Hospital of Xuzhou Medical University, Xuzhou 221002 Jiangsu, China

Correspondence: Hai-Lei Ding (haileimdar@yahoo.com) or Jun-Li Cao (caojl0310@aliyun.com)

These authors contributed equally: Di Liu, Qian-Qian Tang, Di Wang
}

Received: 20 February 2020 Revised: 30 April 2020 Accepted: 12 May 2020

Published online: 19 May 2020 
BDNF expression within the hippocampus and the prefrontal cortex [32-35]. Clinical study also shows that BDNF serum concentrations in depressed patients are increased during lithium augmentation of antidepressants [36]. Taken together, these findings suggest that BDNF may play essential roles in contributing to the therapeutic properties of lithium. However, it remains unclear whether the pathophysiological functions of BDNF within the mesocorticolimbic circuits are involved in the antidepressive actions of lithium.

Using projection-specific electrophysiological recordings, chemogenetics, molecular, and behavioral approaches, our study detects modulatory effects of lithium on the MPFC- and NAcprojecting VTA DA neurons in a CMS-induced mouse model of depression. Furthermore, we demonstrate that the antidepressivelike effects of lithium are mediated by BNDF signaling in the mesocortical DA circuit. These findings reveal circuit-based mechanisms underlying the antidepressive-like effects of lithium and may provide new insights into the neural circuit mechanisms of lithium therapy.

\section{MATERIALS AND METHODS}

Animals

Adult male C57BL/6J mice, around 7-weeks of age, were supplied by the Experimental Animal Center of Xuzhou Medical University, China. Mice were maintained with unrestricted access to food and water on a $12 \mathrm{~h}$ light cycle, and experiments were conducted during the light portion. Animals were housed 4-5 per cage and randomly assigned into experimental groups. All procedures were conducted in accordance with the U.S. National Institutes of Health Guide for the Care and Use of Laboratory Animals.

Chronic unpredictable mild stress protocol

Chronic unpredictable mild stress (CMS) was administered to each cohort of mice in a weekly-rotating schedule according to the previously described method [16]. In brief, mice were subjected to 5 consecutive weeks of CMS. The CMS protocols consisted of fasting ( $24 \mathrm{~h}$ ), water deprivation $(24 \mathrm{~h})$, cage tilted $45^{\circ}$ overnight, tail pinching ( $3 \mathrm{~min}, 1.5 \mathrm{~cm}$ from the tail tip), $4{ }^{\circ} \mathrm{C}$ cold bath $(5 \mathrm{~min})$, damp bedding ( $200 \mathrm{ml}$ water in padding), and day/night inversion, with 5-7 stressors involved randomly each week.

\section{Drugs}

Clozapine-N-oxide (CNO) was dissolved in dimethyl sulfoxide (DMSO) and diluted with $0.9 \%$ saline to a final concentration of $5 \mu \mathrm{M}$ CNO and $0.001 \%$ DMSO. $0.3 \mu \mathrm{l}$ CNO was infused into the VTA to generate in vivo manipulation of dopamine neurons in mice expressing AAV2/9-DIO-hM4Di(Gi)-mCherry or AAV2/9-DIO$\mathrm{hM} 3 \mathrm{Dq}(\mathrm{Gq})-\mathrm{mCherry}$. Mice received $37.5 \mathrm{mg} / \mathrm{L}, 75 \mathrm{mg} / \mathrm{L}, 150 \mathrm{mg} / \mathrm{L}$, $300 \mathrm{mg} / \mathrm{L}, 600 \mathrm{mg} / \mathrm{L}$, or $1200 \mathrm{mg} / \mathrm{L}$ of lithium chloride (SigmaAldrich) in drinking water for 14 days (henceforth referred to as $\mathrm{Li}^{+} 37.5-, \mathrm{Li}^{+} 75-, \mathrm{Li}^{+} 150-, \mathrm{Li}^{+} 300-, \mathrm{Li}^{+} 600-$, or $\mathrm{Li}^{+} 1200$-treated mice). This procedure was chosen based on drug effectiveness and results from previous studies [37, 38]. Controls were given normal drinking water.

\section{Stereotactic surgeries}

Animals were anesthetized with $1.5 \%$ sevoflurane at an oxygen flow rate of $1 \mathrm{~L} / \mathrm{min}$ (for single microinjection or cannula implant), and mounted on a stereotaxic apparatus (Stoelting Co, Wooddale, IL). Eyes were lubricated with an ophthalmic ointment. The fur was shaved, and the incision site was sterilized prior to surgical procedures. Mouse skull surface was exposed, and lumafluor, chemicals or virus were unilaterally microinjected with 33-gauge needle in the MPFC, NAc shell or VTA at the following coordinates (in millimeter): $\mathrm{mPFC}$ (anterior-posterior, +1.8; lateral-medial, 0.4; dorsal-ventral, -2.5), or NAC shell (anterior-posterior, +1.6; lateral-medial, 0.8; dorsal-ventral, -4.5), VTA (anterior-posterior,
-3.5; lateral-medial, 0.5; dorsal-ventral, -4.0). Microinjection was performed in a volume of $0.15-0.3 \mu \mathrm{l}$ at a rate of $0.1 \mu \mathrm{l} / \mathrm{min}$ followed by $5 \mathrm{~min}$ of rest for lumafluor, chemicals, or virus to diffuse. The green and red retrograde beads were purchased from Lumafluor Inc (Lumafluor Inc, Durham, NC). Adeno-associated viruses AAV2/9-TH-Cre-SV40-WPRE (AAV2/5-Cre), AAV2/9-DIOhM3Dq(Gq)-mCherry-WPREs-pA and AAV2/9-DIO-hM4Di(Gi)mCherry-WPREs-pA or its control AAV2/9-DIO-mCherry-WPREs-pA (BrainVTA, Wuhan, China) were prepared at titers of $\sim 2-5 \times 10^{12}$ $\mathrm{vg} / \mathrm{ml}$. For behavioral tests, mice received an intra-mPFC injection of TrkB-Fc (20 ng/0.2 $\mu \mathrm{L}$, T8694; Sigma-Aldrich), or its control IgGFc $[16,39]$. All drugs were diluted in PBS and injected through the cannula at a rate of $0.1 \mu \mathrm{l} / \mathrm{min}$ followed by $5 \mathrm{~min}$ of rest. The detailed timeline of microinjections and behavior tests for each experiment were presented in each figure and figure legend accordingly.

\section{Behavioral tests}

For all behavior tests, mice were allowed to acclimatize in temperature-controlled $\left(25^{\circ} \mathrm{C}\right)$ and noise-free testing room for $1 \mathrm{~h}$ before testing. The mice were only used for a given behavioral task. All of the tests were conducted in a blinded manner.

Tail suspension test (TST)

The TST was conducted as previously described methods [16]. Animals were suspended $50 \mathrm{~cm}$ above the floor padding. The immobility time for $5 \mathrm{~min}$ was calculated.

\section{Sucrose preference test (SPT)}

The mouse was placed (5:00 p.m.) in individual cages, with ad libitum access to $1 \%$ sucrose solution or tap water in 2 bottles $(50 \mathrm{~mL})$ fitted with ball-point sipper tubes [16]. To obviate side preference, the position of the bottles was interchanged every $12 \mathrm{~h}$, with the liquids weighed at the end of the experiment (5:00 p.m. the next day). Sucrose preference was calculated as a percentage of sucrose solution intakes relative to total consumption for a $24 \mathrm{~h}$ duration.

\section{Statistical analyses}

Data were expressed as mean \pm SEM or box and whiskers plot. Normality was tested and statistics were analyzed with GraphPad Prism 6.0. Biochemical, electrophysiological, and behavioral data were analyzed with one-way ANOVA followed by Tukey's post hoc test or Bonferroni's post hoc test. Two-way repeated-measures ANOVA was used to compare the behavioral data from different time points with Tukey's post hoc test. The sample sizes in each experiment were determined based on previous experience to yield sufficient testing power. The criterion for statistical significance was $p<0.05$.

\section{RESULTS}

Lithium differentially regulates the firing rates of $\mathrm{MPFC}$ - and NAcprojecting VTA DA neurons in CMS mice

Given the important roles of the VTA-mPFC and VTA-NAc circuits in regulating depressive-like behaviors [13-18], we investigated whether these two projections were regulated by lithium. To label the VTA-mPFC and VTA-NAc neurons, retrograde lumafluor was injected into the MPFC or NAc shell (Fig. S1A). Immunofluorescence staining revealed that the majority of the VTA-mPFC and VTA-NAc cells were DA neurons (Fig. S1B). Previous study shows $<1 \%$ overlap of VTA DA neurons that project to both the mPFC and NAC [14]. Under our experimental conditions, we detected similarly low overlapping rate ( 2\%; Fig. S1C-E), indicating that mPFC- and NAc-projecting DA neurons were two separate subpopulations of neurons in the VTA. Using projection-specific labeling, we next measured the percentage changes in spontaneous firing rates of MPFC- and NAc-projecting VTA DA neurons in 
mPFC-projecting VTA DA neurons
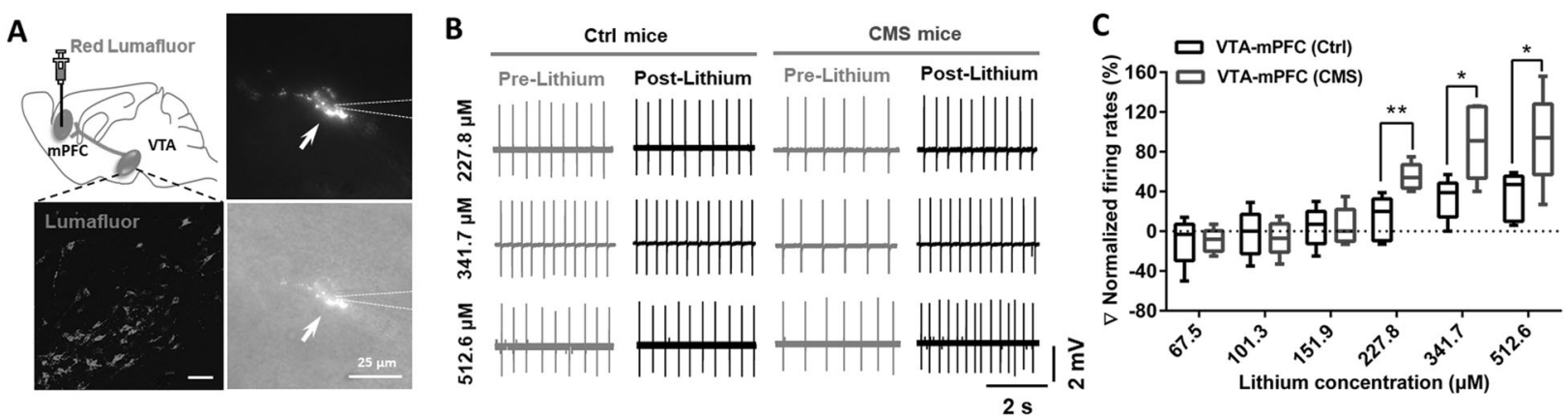

NAc shell-projecting VTA DA neurons
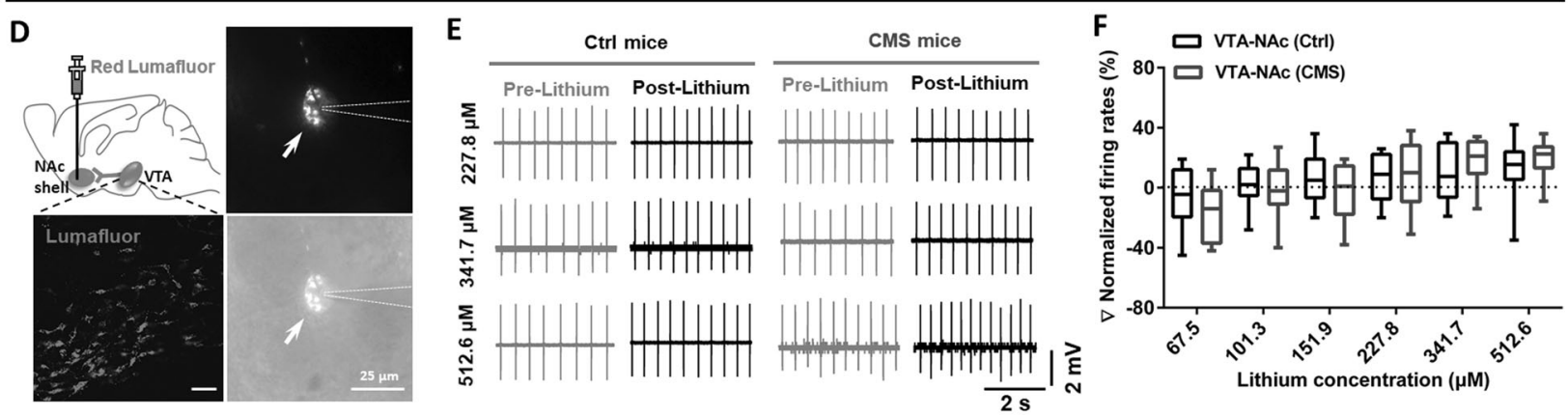

Fig. 1 Lithium differentially regulated the firing activity of mPFC- and NAc-projecting VTA DA neurons in CMS mice. a Confocal images (left) showing labeling of VTA-mPFC neurons and IR-DIC images (right) of a lumafluor-expressing VTA neuron (arrow) and the glass peptide (dashed lines) during electrophysiological recording. Scales, $50 \mu \mathrm{m}$ (left), $25 \mu \mathrm{m}$ (right). b Representative traces showing in vitro spontaneous firings of mPFC-projecting VTA DA neurons before (pre-lithium) and after (post-lithium) bath application of lithium at different concentrations $(227.8,341.7$, and $512.6 \mu \mathrm{M})$. c Bath administration of hight-doses lithium $(227.8,341.7$, and $512.6 \mu \mathrm{M})$ in VTA slices induced dramatic increment in the firing rates of VTA-mPFC dopamine neurons in mice under depressive-like status $\left({ }^{*} p<0.05 ;{ }^{*} p<0.01\right.$ vs. VTA-mPFC (Ctrl) group; $n=4$ animals/5 neurons per group). d Confocal images (left) showing labeling of VTA-NAc neurons and IR-DIC images (right) of a lumafluor-expressing VTA neuron (arrow) and the glass peptide (dashed lines) during electrophysiological recording. Scales, $50 \mu \mathrm{m}$ (left), $25 \mu \mathrm{m}$ (right). e Representative traces showing in vitro spontaneous firings of NAc shell-projecting VTA DA neurons before (pre-lithium) and after (post-lithium) bath application of lithium at different concentrations (227.8, 341.7, and 512.6 $\mu \mathrm{M}$ ). $\mathbf{f}$ Bath administration of lithium in VTA slices could not induce significant changes in the firing activity of VTA-NAc DA neurons in mice under depressive-like status (Unpaired $t$ test, $p>0.05$ vs. VTA-NAc (Ctrl) group; $n=6$ animals/8 neurons per group). The box represents the 25-75th percentiles. Whiskers represent minimum and maximum. See also Fig. S1.

response to bath-applied lithium (from 67.5 to $512.6 \mu \mathrm{M}, 1.5$ times for each stepwise increase) in control and CMS mice (Fig. 1a, d). Results showed that $227.8 \mu \mathrm{M}$ (unpaired $t$ test $\left[t_{8}=3.640, p=\right.$ $0.0066]$ ), $341.7 \mu \mathrm{M}$ (unpaired $t$ test $\left[t_{8}=2.970, p=0.0179\right]$ ), and $512.6 \mu \mathrm{M}$ (unpaired $t$ test $\left[t_{8}=2.473, p=0.0385\right]$ ) lithium increased the firing rates in MPFC-projecting VTA DA neurons in CMS mice (Fig. 1b, c). However, bath application of lithium at different dosages was insufficient to change the firing activity of the NAc-projecting VTA DA neurons in both stress-naïve control mice and CMS mice (Fig. 1e, f). These findings suggested that lithium's regulation on the firing activity of VTA DA neurons displayed the concentration-, state-, and circuit-dependent properties.

High-dose lithium restores the firing rates of mPFC-projecting VTA DA neurons and relieves depressive-like behaviors

Next, we tested the effects of chronic treatment with different doses of lithium on depressive-like behaviors in CMS mice (Fig. 2a). The high-doses lithium used in our study results in brain lithium levels of $\sim 0.8-1 \mathrm{mM}$, which is comparable to the therapeutic levels in humans $[38,40,41]$. Chronic administration of high-doses (600 and $1200 \mathrm{mg} / \mathrm{L})$, but not low-doses $(37.5-300 \mathrm{mg} / \mathrm{L}$; dissolved in drinking water for 14 days), lithium produced an antidepressivelike effect, as evidenced by decreased immobility time in the TST (one-way ANOVA $\left[F_{8,63}=8.995, p<0.0001\right]$ ) and increased sucrose consumption in the SPT (one-way ANOVA $\left[F_{8,63}=7.504\right.$, $p<0.0001$ ]) in CMS mice (Fig. 2b, c). Furthermore, electrophysiological recording in VTA slices showed that the MPFC-projecting VTA DA neurons exhibited a $\sim 60 \%$ decrease in the firing rates in CMS mice when compared with stress-naïve control mice. This decrease was restored in CMS mice administrated with high- $(600 \mathrm{mg} / \mathrm{L})$, but not low- $\left(75 \mathrm{mg} / \mathrm{L}\right.$ ), dose of lithium (one-way ANOVA $\left[F_{5,40}=7.859\right.$, $p<0.0001]$; Fig. $2 \mathrm{~d}$, e). In contrast, these differences were not observed in the NAc-projecting VTA DA neurons (one-way ANOVA $\left[F_{5,37}=0.2774, p=0.9226\right]$; Fig. 2f, g). Collectively, these data confirmed the importance of increased firing rates of VTA-mPFC DA neurons for promoting alleviation of depression-like behavioral abnormalities in CMS mice received chronic administration of highdose lithium.

The increase in the firing activity of mPFC-, but not NAc-, projecting VTA DA neurons mimics the antidepressive-like effects of lithium

Given that the firing activity of mPFC-projecting VTA DA neurons was inhibited in CMS mice and high-dose lithium could restore this inhibition and produce antidepressive-like effects, it was reasonable to speculate that regulation of firing activity in the mPFC-projecting VTA DA neurons was required for the antidepressive actions of high-dose lithium. Therefore, we first examined whether activation of mPFC-projecting VTA DA neurons with chemogenetic approach was antidepressive. To selectively targeted VTA DA neurons that project to the MPFC, we injected a 
A

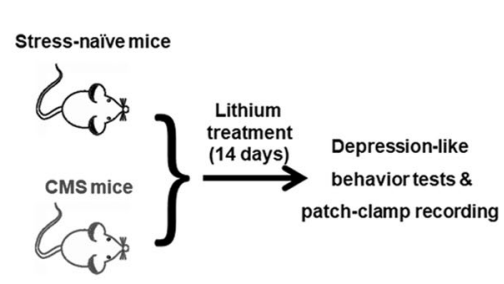

B

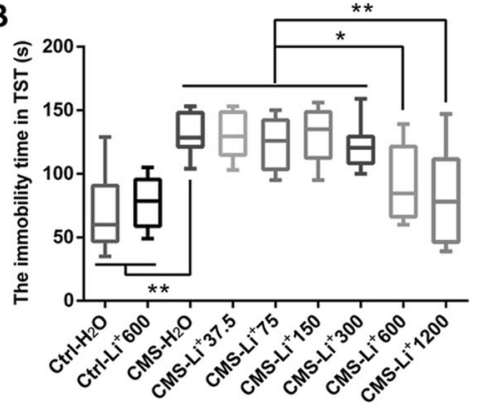

C

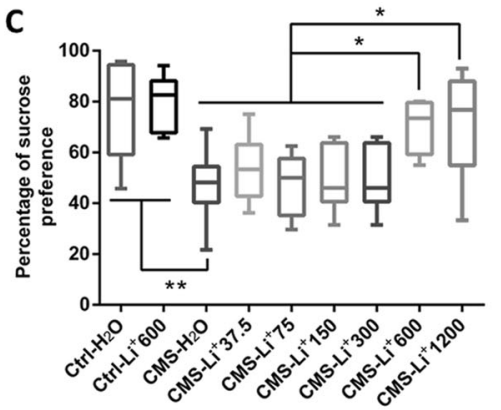

D
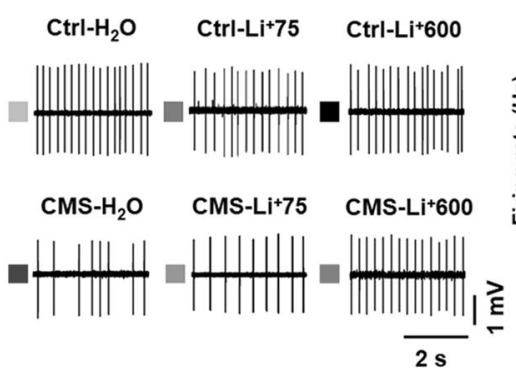

$\mathbf{E}$

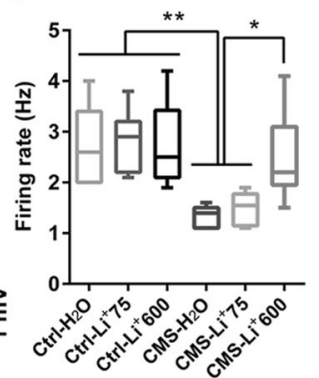

$\mathbf{F}$

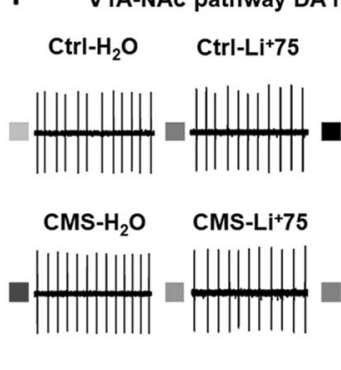

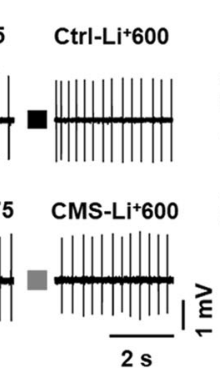

$\mathbf{G}$

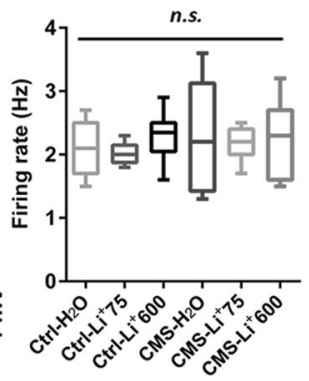

Fig. 2 Chronic treatment of high-dose lithium relieved depressive-like behaviors and increased the firing activity in VTA-mPFC DA neurons in CMS mice. a Schematic showing the experimental design for studying the effects of chronic lithium treatment on the depressivelike behaviors in CMS mice. b High-dose but not low-dose lithium treatment decreased the immobility time in CMS mice in the tail suspension test (TST) $\left({ }^{*} p<0.05 ;{ }^{* *} p<0.01 ; n=8\right.$ mice/group). c High-dose but not low-dose lithium increased the $1 \%$ sucrose preference of CMS mice in the sucrose preference test $\left({ }^{*} p<0.05 ;{ }^{* *} p<0.01 ; n=8\right.$ mice/group). d Sample traces showing in vitro spontaneous firing of VTA-mPFC DA neurons in VTA slices. e Comparison of spontaneous firing in putative VTA-mPFC DA neurons from stress-naïve control and CMS mice that were subjected to chronic lithium treatment $(0,75$, and $600 \mathrm{mg} / \mathrm{L})\left({ }^{*} p<0.05 ;{ }^{*} p<0.01 ; n=6\right.$ animals/7-9 neurons per group). f, $\mathbf{g}$ Sample traces (f) and comparison (g) of in vitro spontaneous firing rates of VTA-NAc DA neurons in VTA slices of stress-naïve control and CMS mice that were subjected to chronic lithium treatment $(0,75$, and $600 \mathrm{mg} / \mathrm{L})(n=4$ animals $/ 6-8$ neurons per group). n.s. not significant. The box represents the $25-75$ th percentiles and the whiskers represent minimum and maximum.

retrograde AAV-TH (Tyrosine Hydroxylase)-Cre into the mPFC and Cre-dependent AAV-DIO-mCherry or AAV-DIO-hM3Dq(Gq)mCherry into the VTA (Figs. 3a and S2A). In VTA slices prepared from these mice, the mCherry-positive neurons also expressed $\mathrm{TH}$ (Fig. 3a). Furthermore, slice recordings showed that bath application of CNO $(5 \mu \mathrm{M})$ increased the spontaneous firing activity in a hM3Dq(Gq)-mCherry-positive VTA neuron (Fig. 3b). To specifically activate the mPFC-projecting VTA DA neurons, CNO $(0.15 \mu \mathrm{l}, 5 \mu \mathrm{M})$ was micro-infused into the VTA in control mice or CMS mice expressed AAV-DIO-mCherry or AAV-DIO-hM3Dq(Gq)mCherry, and infusion of its vehicle was used as a control. The depression-like behavioral tests were measured at $1 \mathrm{~h}$ after CNO injection (Fig. 3c). Results showed that the CMS mice expressed AAV-DIO-hM3Dq(Gq)-mCherry exhibited a decreased immobility time in TST (two-way RM ANOVA; group $\times$ time interaction $\left[F_{3,28}=\right.$ 3.791, $p=0.0212]$; main effect group $\left[F_{3,28}=28.23, p<0.0001\right]$; Fig. $3 d$ ) and increased sucrose consumption in SPT (two-way RM ANOVA; group $\times$ time interaction $\left[F_{3,28}=1.167, p=0.3397\right.$; main effect group $\left[F_{3,28}=12.89, p<0.0001\right]$; Fig. 3e) when compared with those expressed AAV-DIO-mCherry, suggesting that activation of mPFC-projecting VTA DA neurons ameliorated the depression-like behaviors in CMS mice. This effect was not seen in CMS mice with DMSO injection (Fig. S2B-D).

The VTA-NAC DA pathway has also been implicated in stress responses [14], we thus carried out experiments to investigate the roles of this pathway in promoting antidepressive-like effects observed in this study. We first injected a retrograde traveling AAV-TH-Cre into the NAC shell and the Cre-dependent AAV-DIOmCherry or AAV-DIO-hM3Dq(Gq)-mCherry into the VTA (Figs. If and S3A). Immunohistochemical and electrophysiological validation confirmed the viability of using $\mathrm{TH}$-Cre to express functional hM3Dq in VTA-NAc DA neurons (Fig. $3 f$, g). We next tested the effect of activating VTA-NAc DA neurons on depressive-like behaviors in CMS mice (Fig. 3h). Results showed that this manipulation was insufficient to change the immobility time (two-way RM ANOVA; group $\times$ time interaction $\left[F_{3,28}=0.3332, p=\right.$ 0.8014]; main effect group $\left[F_{3,28}=65.24, p<0.0001\right]$; Fig. 3i) and sucrose consumption (two-way RM ANOVA; group $\times$ time interaction $\left[F_{3,28}=0.1922, p=0.9088\right]$; main effect group $\left[F_{3,28}=11.17\right.$, $p<0.0001]$; Fig. 3j) in CMS mice when compared with those expressed AAV-DIO-mCherry. The depressive-like phenotypes were also not changed in CMS mice with DMSO injection (Fig. S3B-D). These results further confirmed the critical roles of the mesocortical DA circuit in mediating CMS-induced depressivelike behaviors.

The increase in the firing activity of mPFC-projecting VTA DA neurons is required for the antidepressive-like effects of high-dose lithium

Next, to explore whether upregulation of firing activity in mPFCprojecting VTA DA neurons was required for the antidepressive actions of high-dose lithium, we injected AAV-TH-Cre into the mPFC and AAV-DIO-mCherry or AAV-DIO-hM4Di(Gi)-mCherry into the VTA in the CMS mice received chronic lithium treatment $(600 \mathrm{mg} / \mathrm{L})$ (Figs. 4a and S4A). Immunohistochemistry verified that 93\% of the mCherry-positive neurons in the VTA expressed TH and projected to the mPFC with terminals spreading around MPFC neurons (Fig. 4a-c). Furthermore, slice recordings confirmed decreased spontaneous action potentials in a hM4Di(Gi)mCherry-positive VTA neuron by bath application of CNO $(5 \mu \mathrm{M})$ (Fig. 4d). Behavioral results showed that inhibition of mPFCprojecting VTA DA neurons via intra-VTA injection of CNO abolished the antidepressive-like effects of high-dose lithium in CMS mice (two-way RM ANOVA; for TST: group $\times$ time interaction 
A

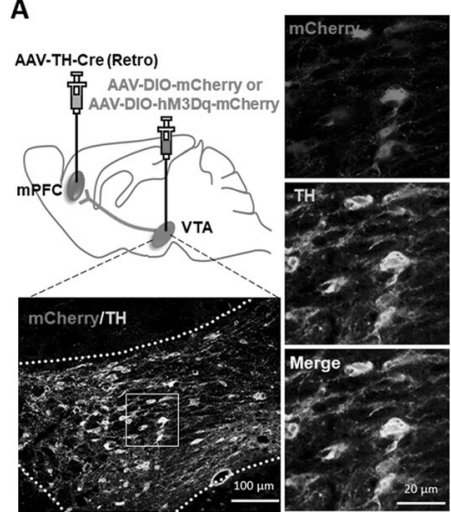

$\mathbf{F}$

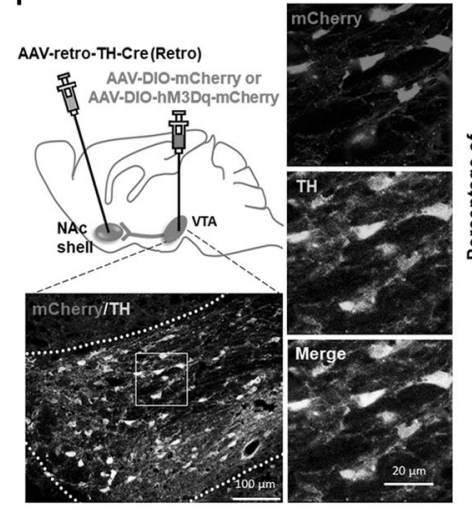

B

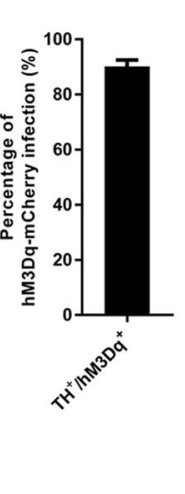

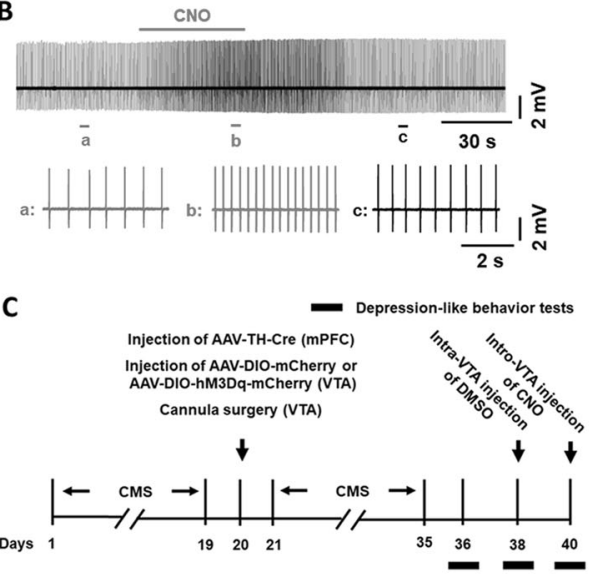

G

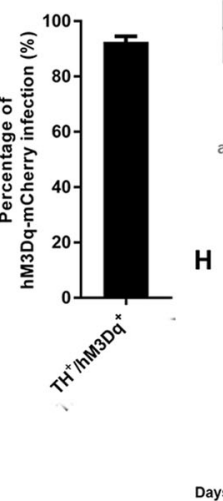

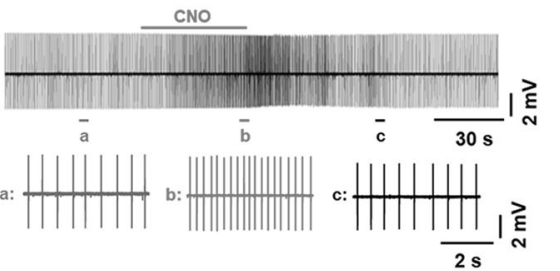

Days

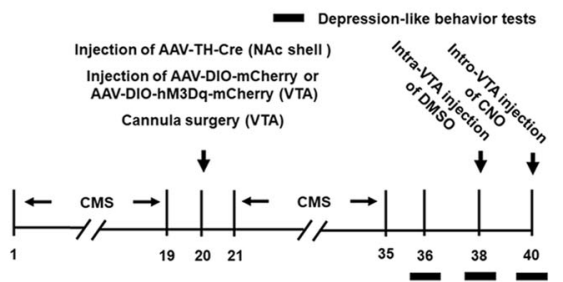

Fig. 3 Chemogenetic activation of VTA-mPFC, but not VTA-NAC, DA neurons rescued depressive-like behaviors in CMS mice. a Schematic showing viral surgeries and confocal image (left) showing expression of AAV-DIO-hM3Dq-mCherry in TH-positive DA neurons from VTA sections. The high magnification images (middle) and quantification (right) showed that $\sim 90.2 \%$ of hM3Dq-mCherry-expressing neurons were TH positive in the VTA ( $n=10$ sections from five animals). Scales, $100 \mu \mathrm{m}$ (left), $20 \mu \mathrm{m}$ (middle). b Cell-attached recordings from an AAV-DIOhM3Dq-mCherry infected VTA-mPFC DA neuron in slice exhibited notable increment in neuronal activity after bath application of CNO (5 $\mu$ M). c Timeline of behavioral experiments. d, e The tail suspension test (d) and sucrose preference test (e) showed that specific activation of the VTA-mPFC DA neurons rescued depressive-like phenotypes in CMS mice $\left({ }^{*} p<0.05 ;{ }^{* *} p<0.01 ;{ }^{* *} p<0.001 ; n=8\right.$ mice/group). f Schematic showing viral surgeries, confocal images (left and middle), and quantification (right) showing co-expression of hM3Dq-mCherry and TH in the VTA. Scales, $100 \mu \mathrm{m}$ (left), $20 \mu \mathrm{m}$ (middle). g Bath application of CNO $(5 \mu \mathrm{M})$ significantly increased the firing activity in an AAV-DIO-hM3Dq$m$ Cherry infected VTA-NAc DA neuron during cell-attached recordings. $\mathbf{h}$ Timeline of behavioral experiments. $\mathbf{i}$, $\mathbf{j}$ Chemogenetic activation of the VTA-NAc DA neurons did not change depressive-like behaviors in CMS mice, as shown by the tail suspension test (i) and sucrose preference test (j) $\left({ }^{*} p<0.05 ;{ }^{* *} p<0.01 ;{ }^{* * *} p<0.001\right.$ vs. either Ctrl-mCherry or Ctrl-hM3Dq group; $p>0.05$ vs. CMS-mCherry group; $n=8$ mice/ group). n.s. not significant. The box represents the $25-75$ th percentiles. Whiskers represent minimum and maximum. See also Figs. S2 and S3.

$\left[F_{6,56}=3.972, p=0.0022\right] ;$ main effect group $\left[F_{3,28}=7.773, p<\right.$ 0.001]; for SPT: group $\times$ time interaction $\left[F_{6,56}=4.933, p=0.0004\right]$; main effect group $\left[F_{3,28}=7.287, p<0.001\right]$; Fig. $\left.4 \mathrm{e}-\mathrm{g}\right)$. The antidepressive-like effects of high-dose lithium in these mice were not changed after micro-infusion of DMSO, the vehicle control for CNO, into the VTA (Fig. S4B-D). These results suggested the VTA-mPFC DA projection as a key circuit basis for the antidepressive-like effects of high-dose lithium.

BDNF signaling in VTA-mPFC circuit mediates the antidepressivelike effects of high-dose lithium

Multiple lines of evidences have demonstrated that BDNF signaling in mesolimbic circuitry regulates depressive-like behaviors under chronic stress [28-31]. Our recent study also showed that intra-mPFC injection of exogenous BDNF attenuated depressive-like behaviors, and blocking BDNF signaling in mPFC prevented the relief of depressive-like behaviors induced by VTA DA neuronal activation in CMS mice [16]. These results lead us to hypothesize that BDNF signaling in the VTA-mPFC DA circuit may mediate the antidepressive-like effects of high-dose lithium. To test this hypothesis, ELISA assay and western blot analysis were used to measure BDNF protein levels in the MPFC, and FISH was conducted to detect $B d n f$ mRNA expression in the VTA in CMS mice treatment with high $(600 \mathrm{mg} / \mathrm{L})$ and low $(75$ $\mathrm{mg} / \mathrm{L}$ ) dose lithium (Fig. 5a). We found that CMS downregulated BDNF protein expression in the $\mathrm{mPFC}$, which was reversed by high-dose (one-way ANOVA $\left[F_{3,16}=9.628, p=0.0007\right]$; Fig. $5 c$ ), but not low-dose lithium (one-way ANOVA, $\left[F_{3,8}=5.815, p=\right.$ 0.0208]; Fig. 5b). Furthermore, ELISA assay showed that BDNF release in the cultured $\mathrm{MPFC}$ slices was decreased in CMS mice, which was restored by high-dose lithium (one-way ANOVA, $\left[F_{3,16}=6.203, p=0.0053\right]$; Fig. $\left.5 d\right)$. However, these effects were not seen in the NAc in CMS mice treated with high-dose lithium (Fig. S5A-C; one-way ANOVA, $\left[F_{3,12}=0.2851, p=0.8352\right]$, Fig. S5B; one-way ANOVA, $\left[F_{3,16}=0.6943, p=0.5688\right]$, Fig. S5C), indicating that lithium's modulation of BDNF expression in the VTA DA neurons displayed the circuit-specific property under depression state. 

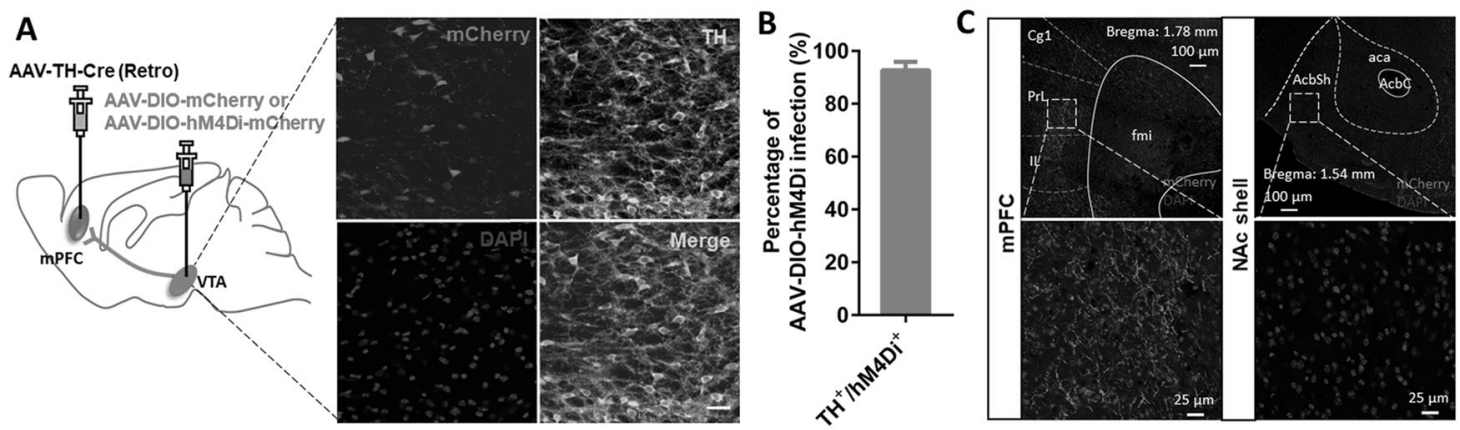

D

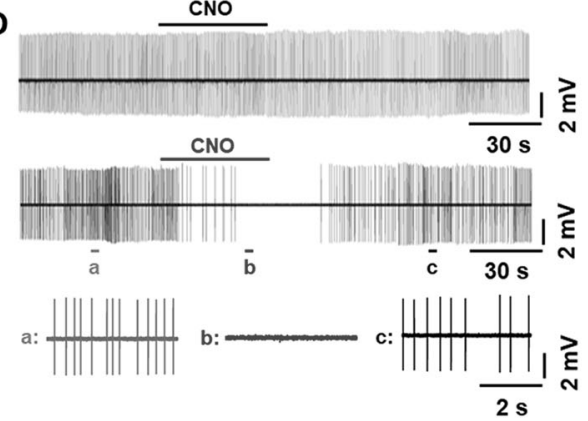

$\mathbf{F}$

$$
\square \text { CMS-Li }{ }^{+} 600+\text { mCherry } \square \text { CMS-Li+ } 600+\text { hM4Di }
$$

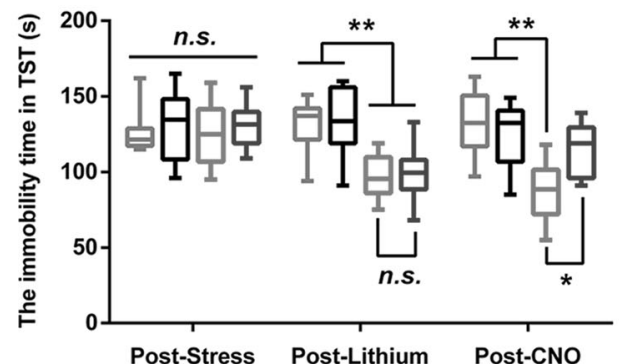

$\mathrm{E}$

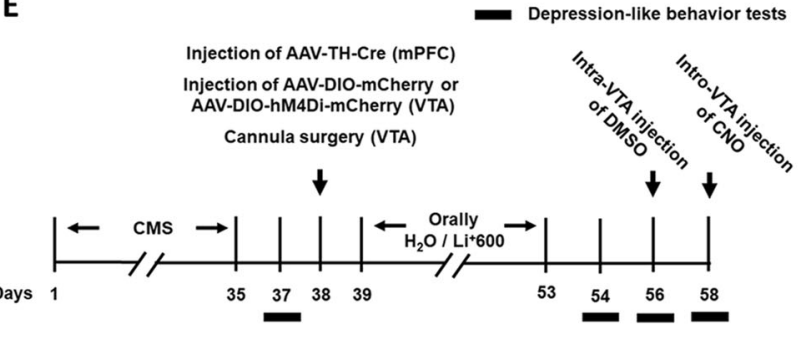

G

$\square$ CMS-H $\mathrm{H}_{2} \mathrm{O}+$ mCherry $\square \mathrm{CMS}-\mathrm{H}_{2} \mathrm{O}+\mathrm{hM} 4 \mathrm{Di}$
$\mathrm{CMS}-\mathrm{Li}^{+} 600+$ mCherry $\square \mathrm{CMS}-\mathrm{Li}^{+} 600+\mathrm{hM} 4 \mathrm{Di}$

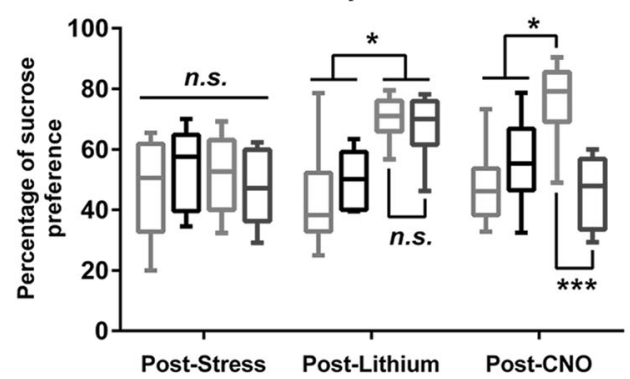

Fig. 4 VTA-mPFC DA circuit contributed to the antidepressive-like effects of high-dose lithium in CMS mice. a Confocal image showing coexpression of AAV-DIO-hM4Di-mCherry in TH-positive DA neurons from VTA sections. Scale, $20 \mu \mathrm{m}$. b Quantification shows that $\sim 93 \%$ of hM4Di-expressing neurons are TH positive in the VTA ( $n=10$ sections from five animals). c Viral hM4Di expression in projection-specific VTA DA neurons allowed for selective control of VTA-mPFC neurons. The hM4Di expression was confirmed by visualization of fluorescent terminals in the MPFC, but not in NAc shell. d Cell-attached recordings from a mCherry-expression (top) and a hM4Di-mCherry-expression (middle and bottom) VTA-mPFC DA neurons in slices with bath application of CNO $(5 \mu \mathrm{M})$. e Timeline of behavioral experiments. $\mathbf{f}, \mathbf{g}$ The tail suspension test $(\mathbf{f})$ and sucrose preference test $(\mathbf{g})$ showed that chemogenetic inhibition of the VTA-mPFC DA neurons reversed the antidepressive-like effects of high-dose lithium in CMS mice $\left({ }^{*} p<0.05 ;{ }^{* *} p<0.01 ;{ }^{* *} p<0.001 ; n=8\right.$ mice/group). For behavioral data, the box represents the $25-75$ th percentiles and the whiskers represent minimum and maximum. The morphological data are represented as mean \pm SEM. See also Fig. S4.

Previous studies have demonstrated anterograde axonal BDNF transportation within the mesocorticolimbic DA circuitry, and there was a strong association between firing rates in VTA DA neurons and BDNF levels in its target regions $[42,43]$. In the present study, we found that CMS downregulated the expression of Bdnf mRNA within mPFC-projecting VTA neurons, which was reversed by systemic high-dose lithium (Fig. 5e). Importantly, blockage of BDNF signaling by intra-mPFC injection of the BDNF scavenger TrkB-Fc $(20 \mathrm{ng} / 0.2 \mu \mathrm{l})$, but not its control IgG-Fc, was able to abolish the antidepressive-like effects of high-dose lithium in CMS mice (two-way RM ANOVA; for TST: group $\times$ time interaction $\left[F_{6,50}=4.952, p=0.0005\right]$; main effect group $\left[F_{3,25}=13.15, p<0.0001\right]$; for SPT: group $\times$ time interaction $\left[F_{6,48}=3.941, p=0.0028\right]$; main effect group $\left[F_{3,24}=17.29\right.$, $p<0.0001]$; Fig. 5f-h). These results suggested that upregulated BDNF in the VTA-mPFC circuit might serve as a mechanistic cause for the alleviation of depressive-like behaviors by highdose lithium.

\section{DISCUSSION}

The mesocorticolimbic DA system has been critically implicated in a variety of psychiatric disorders [13-18]. Consistent with a previous study [16], we found that VTA DA neurons anatomically send nonoverlapping projections to the MPFC and NAC shell. These two projections play distinct roles in the regulation of stress-induced depression [14, 15, 19-22]. Selectively, manipulation of these two subpopulations of VTA DA neurons reshapes emotional behaviors. For example, activation of the VTA-NAc pathway or inhibition of the VTA-mPFC circuit induces susceptibility to social defeat stress $[14,15]$. Conversely, in the CMSinduced depression model, photoactivation of VTA DA neurons rescues depressive-like phenotypes and selective inhibition of VTA DA neurons induces depressive-like behaviors [17]. Interestingly, in present study, we observed that chemogenetic activation of VTA-NAc pathway in control or CMS mice could not change depressive-like behaviors, which is partly consistent with the finding that optogenetic activation of VTA-NAC DA neurons is 
A

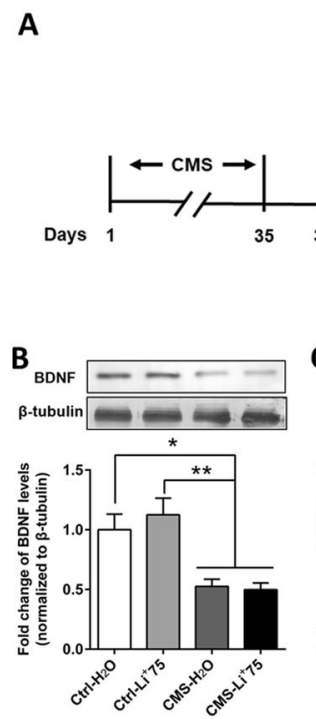

$\mathbf{F}$

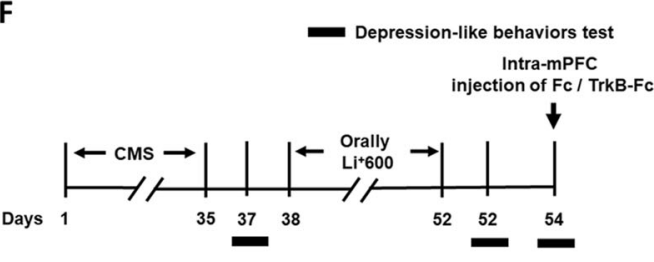

3. VTA Bdnf FISH

\section{$\downarrow$}

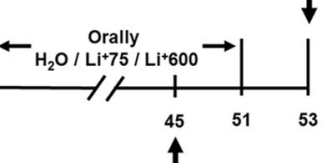

Intra-mPFC injection of retrograde
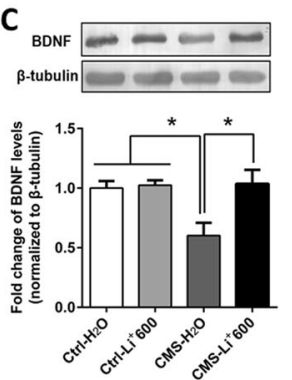

1. $\mathrm{MPFC}$ punches for BDNF protein expression assay

2. MPFC slice for BDNF ELISA

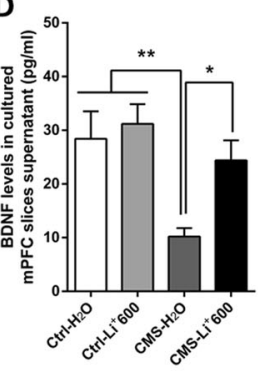

G $\square$ Ctrl-Li+600+FC
$\square$ CMS-Li+600+Fo CMS-Li+600+Fc $\square$ CMS-Li ${ }^{+} 600+$ TrkB-FC

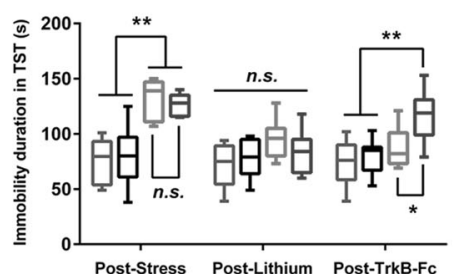

E

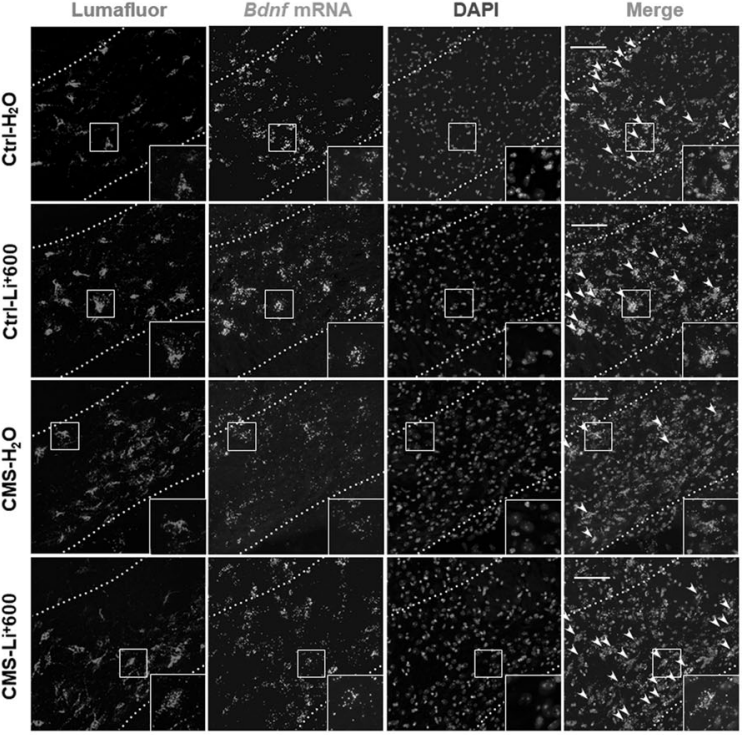

H $\square$ Ctrl-Li $600+$ Fc $\square$ Ctrl-Li' $600+$ TrkB-Fc

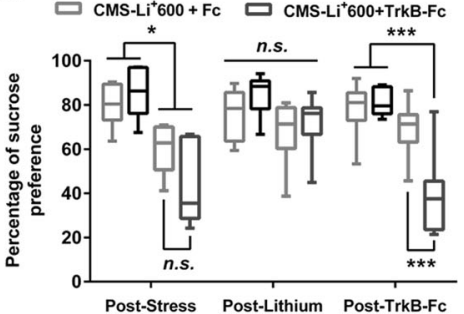

Fig. 5 BDNF signaling in the VTA-mPFC circuit mediated the antidepressive-like effects of high-dose lithium in CMS mice. a Experimental timeline for VTA Bdnf mRNA FISH, BDNF protein western blotting, and mPFC slices for BDNF ELISA assay. b Representative bands and quantitative data showing the effects of treatment with low-dose lithium on BDNF expression in the mPFC in CMS mice $\left({ }^{*} p<0.05\right.$ vs. Ctrl- $\mathrm{H}_{2} \mathrm{O}$ group; ${ }^{* *} p<0.01$ vs. Ctrl-Li 75 group; $n=5$ mice/group). c Representative bands and quantitative data showing that decreased BDNF expression in the mPFC of CMS mice was reversed by treatment with high-dose lithium ( ${ }^{*} p<0.05$ vs. CMS- $\mathrm{H}_{2} \mathrm{O}$ group; $n=3$ mice/group). d BDNF release in the mPFC was decreased in CMS mice and was rescued by chronic treatment with high-dose lithium (* $p<0.05$; ${ }^{* *} p<0.01$ vs. CMS- $\mathrm{H}_{2} \mathrm{O}$ group, $n=5$ mice/group). e FISH assay showed a downregulation of Bdnf mRNA (green) in VTA-mPFC neurons (red) in CMS mice, which was normalized by chronic treatment with high-dose lithium. The arrows indicate VTA-mPFC neurons expressing Bdnf mRNA. Scale, $50 \mu \mathrm{m}$. f Experimental timeline for testing the effects of intra-mPFC administration of TrkB-Fc or its control lgG-Fc on the antidepressive-like effects by high-dose lithium in CMS mice. Tail suspension test (g) and sucrose preference test (h) consistently showed that intra-mPFC administration of TrkB-Fc but not its control lgG-Fc reversed the established antidepressive-like effects of high-dose lithium in CMS mice $\left({ }^{*} p<\right.$ $0.05 ;{ }^{* *} p<0.01 ;{ }^{* * *} p<0.001 ; n=7-8$ mice/group). n.s. not significant. For behavioral data, the box represents the $25-75$ th percentiles and the whiskers represent minimum and maximum. For all other experiments, data are represented as mean \pm SEM.

unable to induce alterations in social interaction and sucrose preference in social defeat stress-naive control mice [14]. Agreement with our current results, previous studies also found that CMS did not change the firing activity of the lateral VTA DA neurons that mostly project to the NAc [44, 45]. The differences observed in effects of activating VTA-NAC DA neurons on depressive-like behaviors in CMS versus social defeat mice may due to the type, intensity, and duration of these stressors. CMS model is consisted of a variety of mild but uncontrollable stressors for 4-12 weeks, resulting in development of anhedonia, a clinical core symptom of depression [46]. However, social defeat stress is consisted of more severe and socially relevant stress for 10 days, causing social avoidance and disruption in circadian and sleep behavior in susceptible mice $[14,47]$. Generally, chronic mild stress decreases VTA DA neuronal activity, yet severe social stress increases the activity of these neurons [48-50], indicating that VTA DA neurons may differentially play roles in the mediation of behavioral abnormalities observed in CMS versus social defeat stress model. Collectively, these results suggest that the mesocorticolimbic DA system is highly complex and functionally heterogeneous. Results from the current study move our understanding one step further by showing the differential roles of the
VTA-mPFC and VTA-NAc projections in depression-like behavioral abnormalities.

Previous studies suggest that lithium might exert its therapeutic actions by modulating DA neurotransmission [24-27]. However, results about the effects of lithium on the mesocorticolimbic DA system are inconsistent. For example, some earlier studies report that chronic lithium treatment significantly attenuates potassium-evoked DA release and decreases extracellular DA levels in the NAC [27, 51]. In a genetic Clock- $\Delta 19$ mouse model of mania, chronic lithium treatment ameliorates neurophysiological deficits and decreases DA levels in the NAc [24]. Using the fast-scan cyclic voltammetry technique, another study also shows that chronic lithium treatment decreases DA release in the NAc following electrical stimulation of the VTA [26]. By contrast, some other studies report that chronic lithium administration upregulates the basal activity of NAc and frontal cortices [52], increases the amphetamine-induced DA increase in the NAC [53], and promotes food-motivated behavior by elevating DA output in the NAc and mPFC [54]. These discrepant results may be due to different experimental procedures and approaches. Here, the functional heterogeneity in two separate subpopulations of VTA 
DA neurons demonstrated in our study might provide insights into reconciling some of these results.

A relatively consistent view is that acute lithium treatment does not significantly affect the mesocorticolimbic DA system, including DA release, extracellular DA levels, and DA neuronal activity $[26,51,53]$. In most of the previous studies, the DA level is used as the main index measuring the effects of lithium on the mesocorticolimbic DA system. It has now become possible to perform more specific interrogation of lithium's effects on the mesocorticolimbic DA system. Using circuit-specific electrophysiological recordings, we demonstrate that the firing rates of the mPFC-, but not NAc-, projecting VTA DA neurons in CMS mice showed a significant increase in response to high concentrations of lithium. In parallel, chronic administration of high-dose lithium restored the firing properties of mPFC-projecting DA neurons, and also rescued CMS-induced depressive-like behaviors. Three possible reasons may contribute to the dose- and circuit-specific effects of lithium. First, different subpopulations of VTA DA neurons exhibit intrinsic heterogeneity in expression of receptors those regulate neuronal excitability [20]. These receptors are potential targets for lithium treatment. For example, lithium suppresses VTA function through the glucagon-like-peptide-1 receptor, which exists in only one subpopulation of VTA DA neurons [55]. Second, lithium distribution varies in the central nervous system and changes according to the administered dose $[56,57]$. Lithium therefore governs the upstream nuclei, such as the nucleus of the solitary tract, to influence different subpopulations of VTA DA neurons [58-60]. Third, lithium regulates different intracellular signaling cascades at different doses [61-63], resulting in dose-dependent pathophysiological properties. Continued dissection of the neural mechanisms for stress processing is vital for understanding and treatment of the related neuropsychiatric disorders. Our data demonstrated differential roles of mesocortical and mesolimbic DA projections in depressive-like behaviors and established a circuit foundation for lithium-based antidepressive treatments.

Preclinical studies have provided evidences for the main molecular determinants of lithium's action [9-12]. A growing literature reveals that BDNF might be a key target of lithium's action. Chronic lithium treatment elicits its antimanic effects via hippocampal BDNF-TrkB dependent synaptic downscaling [32-36, 64]. Long-term treatment of lithium increases the intracellular and extracellular BDNF levels and upregulates exon IV-containing Bdnf mRNA expression in primary cultures of cortical and hippocampal neurons $[35,65]$. Clinical studies also find that lithium treatment in depressed patients restored serum BDNF levels $[36,66,67]$. In agreement with these studies, we demonstrate that high-dose lithium exerts its antidepressant-like role through rescuing the decreased neural activity and restoring the down-expressed Bdnf mRNA in VTA-mPFC DA neurons, and thus increases BDNF levels in the mPFC.

It is interesting that chemogenetic activation of mesocortical DA neurons induces the alleviation of depressive-like phenotypes, which is quickly reversed by scavenging BDNF signaling in the mPFC. Likewise, intra-NAc injection of TrkB inhibitor, ANA-12, $1 \mathrm{~h}$ before optical stimulation of VTA-NAc pathway eliminates social avoidance in subthreshold social defeat mice [28]. A potential reason for the acute roles of blocking BDNF signaling in our study is that VTA DA neurons release BDNF in the target regions in an activity-dependent manner [68-70], chemogenetic manipulation of VTA DA neurons may regulate BDNF levels in the MPFC, and thus modulate depressive-like behaviors. BDNF acutely modulates central synaptic transmission and neural activity in cortical cultures [71, 72], acute brain slices [73-75] and in vivo [76]. Therefore, scavenging BDNF signaling might change neuronal activity and result in behavioral responses $[77,78]$. Taken together, these findings suggest that regulation of BDNF signaling in mesocortical DA circuit may be a potential mechanism underlying the antidepressant-like effects of high-dose lithium. A limitation of this study, however, is that the roles of BDNF derived from local $\mathrm{mPFC}$ neurons in the antidepressive effects of lithium require further investigation.

\section{CONCLUSIONS}

Taken together, our findings provide novel insights into the roles of BDNF within VTA-mPFC DA projection in mediating lithium's actions. This study reveals new molecular and circuitry mechanisms underlying the therapeutic effects of lithium, shedding light on the potential to extend lithium's clinical applications.

\section{FUNDING AND DISCLOSURE}

The authors declare no competing interests. This study was supported by the National Natural Science Foundation of China (81720108013, 31771161, 81070888, and 81230025 to JLC, 81200859 to HLD), Jiangsu Province Natural Science Foundation (BK20171159 to HLD), the "Xing-Wei" Project of Jiangsu Province Department of Health (RC2007094, XK201136), the Key Project of Nature Science Foundation of Jiangsu Education Department (11KJA320001 to JLC, 17KJA320005 to HLD), the Jiangsu Provincial Special Program of Medical Science (BL2014029), the Jiangsu Province Ordinary University Graduate Innovation Plan (KYCX19_2228 to Di Wang), and the Priority Academic Program Development of Jiangsu Higher Education Institutions.

\section{ACKNOWLEDGEMENTS}

The authors thank Ms. Cui Yin and Mr. Peng Wu for their technical assistance.

\section{AUTHOR CONTRIBUTIONS}

DL, HLD, and JLC designed the study, DL, QQT, DW, SPS, XNY, ZYW, ZX, HL, and JXY collected the data, DL and SWH performed the surgeries, DL, QQT, and DW analyzed the data, DL and SEM prepared the paper, $\mathrm{HZ}$ and $\mathrm{MHH}$ revised paper critically for important intellectual content.

Publisher's note Springer Nature remains neutral with regard to jurisdictional claims in published maps and institutional affiliations.

\section{REFERENCES}

1. Freeman MP, Freeman SA. Lithium: clinical considerations in internal medicine. Am J Med. 2006;119:478-81.

2. Price LH, Heninger GR. Lithium in the treatment of mood disorders. N Engl J Med. 1994;331:591-8.

3. Young $\mathrm{AH}$. Lithium for long-term treatment of unipolar depression. Lancet Psychiatry. 2017;4:511-2.

4. Undurraga J, Sim K, Tondo L, Gorodischer A, Azua E, Tay KH, et al. Lithium treatment for unipolar major depressive disorder: systematic review. J Psychopharmacol. 2019;33:167-76.

5. Dold M, Kasper S. Evidence-based pharmacotherapy of treatment-resistant unipolar depression. Int J Psychiatry Clin Pract. 2017;21:13-23.

6. Bschor T, Bauer M, Adli M. Chronic and treatment resistant depression: diagnosis and stepwise therapy. Dtsch Arztebl Int. 2014;111:766-75.

7. Nelson JC, Baumann P, Delucchi K, Joffe R, Katona C. A systematic review and meta-analysis of lithium augmentation of tricyclic and second generation antidepressants in major depression. J Affect Disord. 2014;168:269-75.

8. Bauer M, Adli M, Ricken R, Severus E, Pilhatsch M. Role of lithium augmentation in the management of major depressive disorder. CNS Drugs. 2014;28:331-42.

9. Gould TD, Manji HK. Glycogen synthase kinase-3: a putative molecular target for lithium mimetic drugs. Neuropsychopharmacology. 2005;30:1223-37.

10. Kerr F, Bjedov I, Sofola-Adesakin O. Molecular mechanisms of lithium action: switching the light on multiple targets for dementia using animal models. Front Mol Neurosci. 2018;11:297.

11. Alda M. Lithium in the treatment of bipolar disorder: pharmacology and pharmacogenetics. Mol Psychiatry. 2015;20:661-70.

12. Motoi Y, Shimada K, Ishiguro K, Hattori N. Lithium and autophagy. ACS Chem Neurosci. 2014;5:434-42. 
13. Cao JL, Covington HE 3rd, Friedman AK, Wilkinson MB, Walsh JJ, Cooper DC, et al. Mesolimbic dopamine neurons in the brain reward circuit mediate susceptibility to social defeat and antidepressant action. J Neurosci. 2010;30:16453-8.

14. Chaudhury D, Walsh JJ, Friedman AK, Juarez B, Ku SM, Koo JW, et al. Rapid regulation of depression-related behaviours by control of midbrain dopamine neurons. Nature. 2013:493:532-6.

15. Friedman AK, Walsh JJ, Juarez B, Ku SM, Chaudhury D, Wang J, et al. Enhancing depression mechanisms in midbrain dopamine neurons achieves homeostatic resilience. Science. 2014;344:313-9.

16. Liu D, Tang QQ, Yin C, Song Y, Liu Y, Yang JX, et al. Brain-derived neurotrophic factor mediated projection-specific regulation of depressive-like and nociceptive behaviors in the mesolimbic reward circuitry. Pain. 2018;159:175-88.

17. Tye KM, Mirzabekov JJ, Warden MR, Ferenczi EA, Tsai HC, Finkelstein J, et al. Dopamine neurons modulate neural encoding and expression of depressionrelated behaviour. Nature. 2013;493:537-41.

18. Russo SJ, Nestler EJ. The brain reward circuitry in mood disorders. Nat Rev Neurosci. 2013;14:609-25.

19. Barrot $M$. The ventral tegmentum and dopamine: a new wave of diversity. Neuroscience. 2014;282:243-7.

20. Lammel S, Lim BK, Malenka RC. Reward and aversion in a heterogeneous midbrain dopamine system. Neuropharmacology. 2014;76:351-9.

21. Morales M, Margolis EB. Ventral tegmental area: cellular heterogeneity, connectivity and behaviour. Nat Rev Neurosci. 2017;18:73-85.

22. Walsh JJ, Han MH. The heterogeneity of ventral tegmental area neurons: projection functions in a mood-related context. Neuroscience. 2014;282:101-8.

23. Friedman AK, Juarez B, Ku SM, Zhang H, Calizo RC, Walsh JJ, et al. KCNQ channe openers reverse depressive symptoms via an active resilience mechanism. Nat Commun. 2016;7:11671.

24. Coque L, Mukherjee S, Cao JL, Spencer S, Marvin M, Falcon E, et al. Specific role of VTA dopamine neuronal firing rates and morphology in the reversal of anxietyrelated, but not depression-related behavior in the ClockDelta19 mouse model of mania. Neuropsychopharmacology. 2011;36:1478-88

25. Arey RN, Enwright JF 3rd, Spencer SM, Falcon E, Ozburn AR, Ghose S, et al. An important role for cholecystokinin, a CLOCK target gene, in the development and treatment of manic-like behaviors. Mol Psychiatry. 2014;19:342-50.

26. Can A, Frost DO, Cachope R, Cheer JF, Gould TD. Chronic lithium treatment rectifies maladaptive dopamine release in the nucleus accumbens. J Neurochemistry. 2016;139:576-85.

27. Ferrie $L$, Young $A H, M c Q u a d e ~ R$. Effect of lithium and lithium withdrawal on potassium-evoked dopamine release and tyrosine hydroxylase expression in the rat. Int J Neuropsychopharmacol. 2006;9:729-35.

28. Walsh JJ, Friedman AK, Sun $H_{1}$, Heller EA, Ku SM, Juarez B, et al. Stress and CRF gate neural activation of BDNF in the mesolimbic reward pathway. Nat Neurosci. 2014;17:27-9.

29. Wook Koo J, Labonte B, Engmann O, Calipari ES, Juarez B, Lorsch Z, et al. Essentia role of mesolimbic brain-derived neurotrophic factor in chronic social stressinduced depressive behaviors. Biol Psychiatry. 2016;80:469-78.

30. Martinowich $\mathrm{K}$, Manji $\mathrm{H}$, Lu B. New insights into BDNF function in depression and anxiety. Nat Neurosci. 2007;10:1089-93.

31. Berton O. Essential role of BDNF in the mesolimbic dopamine pathway in social defeat stress. Science. 2006;311:864-8.

32. Fukumoto T, Morinobu S, Okamoto Y, Kagaya A, Yamawaki S. Chronic lithium treatment increases the expression of brain-derived neurotrophic factor in the rat brain. Psychopharmacology. 2001;158:100-6.

33. Moore GJ, Cortese BM, Glitz DA, Zajac-Benitez C, Quiroz JA, Uhde TW, et al. A longitudinal study of the effects of lithium treatment on prefrontal and subgenual prefrontal gray matter volume in treatment-responsive bipolar disorder patients. J Clin Psychiatry. 2009;70:699-705.

34. Post RM. Role of BDNF in bipolar and unipolar disorder: clinical and theoretical implications. J Psychiatr Res. 2007;41:979-90.

35. De-Paula VJ, Gattaz WF, Forlenza OV. Long-term lithium treatment increases intracellular and extracellular brain-derived neurotrophic factor (BDNF) in cortical and hippocampal neurons at subtherapeutic concentrations. Bipolar Disord. 2016;18:692-5.

36. Ricken R, Adli M, Lange C, Krusche E, Stamm TJ, Gaus S, et al. Brain-derived neurotrophic factor serum concentrations in acute depressive patients increase during lithium augmentation of antidepressants. J Clin Psychopharmacol. 2013;33:806-9.

37. Pourmohammadi N, Alimoradi $\mathrm{H}$, Mehr SE, Hassanzadeh G, Hadian MR, Sharifzadeh $M$, et al. Lithium attenuates peripheral neuropathy induced by paclitaxel in rats. Basic Clin Pharmacol Toxicol. 2012;110:231-7.

38. Riedl U, Barocka A, Kolem H, Demling J, Kaschka WP, Schelp R, et al. Duration of lithium treatment and brain lithium concentration in patients with unipolar and schizoaffective disorder-a study with magnetic resonance spectroscopy. Biol Psychiatry. 1997;41:844-50.
39. Zhang H, Qian YL, Li C, Liu D, Wang L, Wang XY, et al. Brain-derived neurotrophic factor in the mesolimbic reward circuitry mediates nociception in chronic neuropathic pain. Biol Psychiatry. 2017;82:608-18.

40. Kato T, Inubushi T, Takahashi S. Relationship of lithium concentrations in the brain measured by lithium-7 magnetic resonance spectroscopy to treatment response in mania. J Clin Psychopharmacol. 1994;14:330-5.

41. Kato $T$, Shioiri $T$, Inubushi $T$, Takahashi S. Brain lithium concentrations measured with lithium-7 magnetic resonance spectroscopy in patients with affective disorders: relationship to erythrocyte and serum concentrations. Biol Psychiatry. 1993;33:147-52.

42. Altar CA, Cai N, Bliven T, Juhasz M, Conner JM, Acheson AL, et al. Anterograde transport of brain-derived neurotrophic factor and its role in the brain. Nature. 1997;389:856-60.

43. Altar CA, DiStefano PS. Neurotrophin trafficking by anterograde transport. Trends Neurosci. 1998;21:433-7.

44. Moreines JL, Owrutsky ZL, Grace AA. Involvement of infralimbic prefrontal cortex but not lateral habenula in dopamine attenuation after chronic mild stress. Neuropsychopharmacology. 2017;42:904-13.

45. Lammel S, Ion DI, Roeper J, Malenka RC. Projection-specific modulation of dopamine neuron synapses by aversive and rewarding stimuli. Neuron. 2011;70:855-62.

46. Willner P. The chronic mild stress (CMS) model of depression: history, evaluation and usage. Neurobiol Stress. 2017;6:78-93.

47. Wells AM, Ridener E, Bourbonais CA, Kim W, Pantazopoulos $\mathrm{H}$, Carroll Fl, et al. Effects of chronic social defeat stress on sleep and circadian rhythms are mitigated by kappa-opioid receptor antagonism. J Neurosci. 2017;37:7656-68.

48. Valenti O, Gill KM, Grace AA. Different stressors produce excitation or inhibition of mesolimbic dopamine neuron activity: response alteration by stress preexposure. Eur J Neurosci. 2012;35:1312-21.

49. Moore H, Rose HJ, Grace AA. Chronic cold stress reduces the spontaneous activity of ventral tegmental dopamine neurons. Neuropsychopharmacology. 2001;24:410-9.

50. Valenti O, Lodge DJ, Grace AA. Aversive stimuli alter ventral tegmental area dopamine neuron activity via a common action in the ventral hippocampus. Neurosci. 2011:31:4280-9.

51. Ichikawa J, Dai J, Meltzer HY. Lithium differs from anticonvulsant mood stabilizers in prefrontal cortical and accumbal dopamine release: role of 5-HT(1A) receptor agonism. Brain Res. 2005;1049:182-90.

52. Basselin M, Chang L, Rapoport SI. Chronic lithium chloride administration to rats elevates glucose metabolism in wide areas of brain, while potentiating negative effects on metabolism of dopamine D2-like receptor stimulation. Psychopharmacology. 2006;187:303-11.

53. Baptista T, Teneud L, Contreras Q, Burguera JL, Burguera M, Hernandez L. Effects of acute and chronic lithium treatment on amphetamine-induced dopamine increase in the nucleus accumbens and prefrontal cortex in rats as studied by microdialysis. J Neural Transm Gen Sect. 1993;94:75-89.

54. Gambarana C, Masi F, Leggio B, Grappi S, Nanni G, Scheggi S, et al. Acquisition of a palatable-food-sustained appetitive behavior in satiated rats is dependent on the dopaminergic response to this food in limbic areas. Neuroscience. 2003;121:179-87.

55. Fortin SM, Chartoff EH, Roitman MF. The Aversive Agent Lithium Chloride Suppresses Phasic Dopamine Release Through Central GLP-1 Receptors. Neuropsychopharmacology 2016;41:906-15.

56. Levine S, Saltzman A, Katof B, Meister A, Cooper TB. Lithium distribution in experimental inflammation of brain and spinal cord. Prog Neuropsychopharmacol Biol Psychiatry. 1996;20:1011-7.

57. Ramaprasad S, Ripp E, Pi J, Lyon M. Pharmacokinetics of lithium in rat brain regions by spectroscopic imaging. Magn Reson Imaging. 2005; 23:859-63.

58. Alhadeff AL, Rupprecht LE, Hayes MR. GLP-1 neurons in the nucleus of the solitary tract project directly to the ventral tegmental area and nucleus accumbens to control for food intake. Endocrinology. 2012;153:647-58.

59. Carter ME, Han S, Palmiter RD. Parabrachial calcitonin gene-related peptide neurons mediate conditioned taste aversion. J Neurosci. 2015;35:4582-6.

60. Carter ME, Soden ME, Zweifel LS, Palmiter RD. Genetic identification of a neural circuit that suppresses appetite. Nature. 2013;503:111-4.

61. Bachmann RF, Schloesser RJ, Gould TD, Manji HK. Mood stabilizers target cellular plasticity and resilience cascades: implications for the development of novel therapeutics. Mol Neurobiol. 2005;32:173-202.

62. Li X, Jope RS. Is glycogen synthase kinase-3 a central modulator in mood regulation? Neuropsychopharmacology. 2010;35:2143-54.

63. Park SW, Lee JG, Seo MK, Cho HY, Lee CH, Lee JH, et al. Effects of mood-stabilizing drugs on dendritic outgrowth and synaptic protein levels in primary hippocampal neurons. Bipolar Disord. 2015;17:278-90. 
64. Gideons ES, Lin PY, Mahgoub M, Kavalali ET, Monteggia LM. Chronic lithium treatment elicits its antimanic effects via BDNF-TrkB dependent synaptic downscaling. Elife. 2017;6:e25480.

65. Yasuda S, Liang MH, Marinova Z, Yahyavi A, Chuang DM. The mood stabilizers lithium and valproate selectively activate the promoter IV of brain-derived neurotrophic factor in neurons. Mol Psychiatry. 2009;14:51-9.

66. D'Addario C, Dell'Osso B, Palazzo MC, Benatti B, Lietti L, Cattaneo E, et al. Selective DNA methylation of BDNF promoter in bipolar disorder: differences among patients with BDI and BDII. Neuropsychopharmacology. 2012;37:1647-55.

67. Tunca Z, Ozerdem A, Ceylan D, Yalcin Y, Can G, Resmi H, et al. Alterations in BDNF (brain derived neurotrophic factor) and GDNF (glial cell line-derived neurotrophic factor) serum levels in bipolar disorder: The role of lithium. J Affect Disord. 2014;166:193-200.

68. Guillin O, Diaz J, Carroll P, Griffon N, Schwartz JC, Sokoloff P. BDNF controls dopamine D3 receptor expression and triggers behavioural sensitization. Nature. 2001;411:86-9.

69. Berton O, McClung CA, Dileone RJ, Krishnan V, Renthal W, Russo SJ, et al. Essential role of BDNF in the mesolimbic dopamine pathway in social defeat stress. Science. 2006;311:864-8.

70. Krishnan V, Han MH, Graham DL, Berton O, Renthal W, Russo SJ, et al. Molecular adaptations underlying susceptibility and resistance to social defeat in brain reward regions. Cell. 2007;131:391-404.
71. Levine ES, Dreyfus CF, Black IB, Plummer MR. Brain-derived neurotrophic factor rapidly enhances synaptic transmission in hippocampal neurons via postsynaptic tyrosine kinase receptors. Proc Natl Acad Sci USA. 1995;92:8074-7.

72. Li YX, Zhang Y, Lester HA, Schuman EM, Davidson N. Enhancement of neurotransmitter release induced by brain-derived neurotrophic factor in cultured hippocampal neurons. J Neurosci. 1998;18:10231-40.

73. Goggi J, Pullar IA, Carney SL, Bradford HF. Modulation of neurotransmitter release induced by brain-derived neurotrophic factor in rat brain striatal slices in vitro. Brain Res. 2002;941:34-42.

74. Akaneya Y, Tsumoto T, Kinoshita S, Hatanaka H. Brain-derived neurotrophic factor enhances long-term potentiation in rat visual cortex. J Neurosci. 1997;17:6707-16.

75. Kang H, Schuman EM. Long-lasting neurotrophin-induced enhancement of synaptic transmission in the adult hippocampus. Science. 1995;267:1658-62.

76. Messaoudi E, Bardsen K, Srebro B, Bramham CR. Acute intrahippocampal infusion of BDNF induces lasting potentiation of synaptic transmission in the rat dentate gyrus. J Neurophysiol. 1998;79:496-9.

77. Lobo MK, Covington HE 3rd, Chaudhury D, Friedman AK, Sun H, Damez-Werno D, et al. Cell type-specific loss of BDNF signaling mimics optogenetic control of cocaine reward. Science. 2010;330:385-90.

78. Choe KY, Han SY, Gaub P, Shell B, Voisin DL, Knapp BA, et al. High salt intake increases blood pressure via BDNF-mediated downregulation of $\mathrm{KCC} 2$ and impaired baroreflex inhibition of vasopressin neurons. Neuron. 2015;85:549-60. 